Pure and Applied Mathematics Quarterly

Volume 1, Number 2

(Special Issue: In memory of

Armand Borel, part 1 of 3)

$291-303,2005$

\title{
Monodromy of Hecke-invariant Subvarieties
}

\author{
Ching-Li Chai
}

Dedicated to the memory of Armand Borel

\section{$\S 1$. Introduction}

The main result of this note is that for any primes $\ell \neq p$, the $\ell$-adic monodromy group of the Zariski closure $\overline{\mathcal{H}\left(x_{0}\right)}$ of the $\ell$-adic Hecke orbit of a non-supersingular point $x_{0}$ in $\mathcal{A}_{g}$ over $\overline{\mathbb{F}_{p}}$ is equal to the full symplectic group; see Prop. 4.1. Here $\mathcal{A}_{g}$ denotes the moduli space of $g$-dimensional principally polarized abelian varieties. The proof consists mostly of group theory. It does use some nontrivial information from algebraic geometry, namely Grothendieck's semisimplicity theorem for pure $\mathbb{Q}_{\ell}$-sheaves and the Riemann hypothesis for abelian varieties over finite fields.

Some immediate consequences of the main results are recorded as corollaries in $\S 4$. A generalization from the case of one prime $\ell$ to the set of all primes not equal to $p$ is formulated in Prop. 4.5.4. The method used in this note applies to the reduction of other Shimura varieties as well, with the reductive group in the Shimura input data replaced by the simply connected cover of its derived group.

The group-theoretic argument has an unexpected geometric consequence. Suppose that $Z$ is a smooth, locally closed subset $Z$ of $\mathcal{A}_{g}$ over $\overline{\mathbb{F}_{p}}$ stable under all $\ell$-adic Hecke correspondences, such that the Hecke correspondences operate transitively on the set of irreducible components of $Z$, and the maximal points of $Z$ are

Received February 23, 2005. Partially supported by grants DMS01-00441 and DMS04-00482 from the National Science Foundation, and a grant from the Nederlandse Organisatie voor Wetenschappelijk Onderzoek. 
not supersingular. Then $Z$ is irreducible; see Prop. 4.4. This result is useful for proving the irreducibility of certain subschemes of $\mathcal{A}_{g}$ defined by $p$-adic invariants of the Barsotti-Tate group of the universal abelian scheme; see 4.7 and 4.8. We also prove an "independence of $\ell$ "-type result, that $Z$ is stable under all prime-to- $p$ Hecke correspondences if it is stable under all $\ell$-adic Hecke correspondences for one prime number $\ell \neq p$; see Prop. 4.6.

It is a pleasure to thank E. P. van den Ban and A. Borel for their help with Lemma 3.3, B. Moonen for pertinent and helpful comments, and F. Oort for stimulating discussions on applications of Prop. 4.4. The author would like to thank the referee for a very careful reading and for pointing out an error in $\S 2$. This paper was written during a visit to Utrecht University in November 2002, and I would like to the Mathematisch Instituut of Utrecht University for hospitality. Financial support from the NSF and the NWO are gratefully acknowledged.

\section{$\S 2$. Notations and preliminaries}

(2.1) Throughout this note, we work over an algebraically closed field $k$ of characteristic $p>0$. Let $\ell$ be a prime number, $\ell \neq p$, and let $N \geq 3$ be a natural number relatively prime to $p \ell$. Let $Z$ be a smooth locally closed subvariety of $\mathcal{A}_{g, N}$ over $k$. Here $\mathcal{A}_{g, N}$ denotes the moduli space of $g$-dimensional principally polarized abelian varieties with principal symplectic level- $N$ structure. Assume that $Z$ is stable under all $\ell$-adic Hecke correspondences coming from $\operatorname{Sp}_{2 g}\left(\mathbb{Q}_{\ell}\right)$, and that the $\ell$-adic Hecke correspondences operate transitively on the set of connected components of $Z$. Let $A \rightarrow Z$ be the restriction to $Z$ of the universal abelian scheme. Let $Z^{0}$ be an irreducible component of $Z$, let $\eta=\eta_{Z}$ be the generic point of $Z^{0}$, and let $\bar{\eta}$ be a geometric point of $Z$ above $\eta$.

(2.2) Denote by $\mathrm{T}=\mathrm{T}_{\ell}=A\left[\ell^{\infty}\right]_{\bar{\eta}}$ the $\ell$-adic Tate module of $A_{\eta}$, and let $V=V_{\ell}=$ $T_{\ell} \otimes \mathbb{Q}_{\ell}$. Let $\langle,\rangle_{\ell}: \mathrm{T}_{\ell} \times \mathrm{T}_{\ell} \rightarrow \mathbb{Z}_{\ell}(1)$ be the Weil pairing. Let $G=\operatorname{Sp}\left(V,\langle,\rangle_{\ell}\right)$, considered as an algebraic group over $\mathbb{Q}_{\ell}$. The semisimple group $G$ has a natural $\mathbb{Z}_{\ell^{-}}$ model whose $\mathbb{Z}_{\ell}$-points is $\operatorname{Sp}\left(\mathrm{T}_{\ell},\langle,\rangle_{\ell}\right)$. We choose and fix an isomorphism between $\left(\mathrm{T}_{\ell},\langle,\rangle_{\ell}\right)$ and the standard symplectic pairing on $\mathbb{Z}_{\ell}^{2 g}$, and use it to identify $G$ with $\mathrm{Sp}_{2 g, \mathbb{Q}_{\ell}}$.

(2.3) Let $\rho_{A, \ell}: \pi_{1}\left(Z^{0}, \bar{\eta}\right) \rightarrow \operatorname{Sp}\left(\mathrm{T}_{\ell},\langle,\rangle_{\ell}\right)$ be the $\ell$-adic representation attached to the $\ell$-adic Tate module of $A \rightarrow Z^{0}$. For each $n \in \mathbb{N}$, denote by $\langle,\rangle_{n}:\left(\mathrm{T}_{\ell} / \ell^{n} \mathrm{~T}_{\ell}\right) \times$ $\left(\mathrm{T}_{\ell} / \ell^{n} \mathrm{~T}_{\ell}\right) \rightarrow\left(\mathbb{Z} / \ell^{n} \mathbb{Z}\right)(1)$ the restriction of the Weil pairing to the $\ell^{n}$-torsion points. Let $\rho_{n}: \pi_{1}\left(Z^{0}, \bar{\eta}\right) \rightarrow \mathrm{Sp}\left(\mathrm{T}_{\ell} / \ell^{n} \mathrm{~T}_{\ell},\langle,\rangle_{\ell}\right)$ be the monodromy representation attached to $A\left[\ell^{n}\right] \rightarrow Z^{0}$, the subgroup of $\ell^{n}$-torsion points of $A \rightarrow Z^{0}$. Let $M_{n}=\rho_{n}\left(\pi_{1}(Z, \bar{\eta})\right)$, 
the image of $\rho_{n}$. The finite groups $M_{n}$ form a projective system whose limit $M$ is the monodromy group $\rho\left(\pi_{1}\left(Z^{0}, \bar{\eta}\right)\right)$. The group $M$ is a compact closed subgroup of $G\left(\mathbb{Q}_{\ell}\right)$.

(2.4) For each $n \in \mathbb{N}$, "forgetting the $\ell$-part of the level structure" defines a morphism $\mathcal{A}_{g, \ell^{n} N} \rightarrow \mathcal{A}_{g, N}$ between moduli spaces; this morphism is a principal étale $\mathrm{Sp}_{2 g}\left(\mathbb{Z} / \ell^{n} \mathbb{Z}\right)$-cover. Moreover we have natural morphisms $\mathcal{A}_{g, \ell^{n+1} N} \rightarrow \mathcal{A}_{g, \ell^{n} N}$. Let $\mathcal{A}_{g, \ell^{\infty} N}$ be the projective limit of the $\mathcal{A}_{g, \ell^{n} N}$ 's. It is a principal pro-étale $\operatorname{Sp}_{2 g}\left(\mathbb{Z}_{\ell}\right)$ cover of $\mathcal{A}_{g, N}$. There is a natural action of $\mathrm{Sp}_{2 g}\left(\mathbb{Q}_{\ell}\right)$ on $\mathcal{A}_{g, \ell^{\infty} N}$, extending the action of $\operatorname{Sp}_{2 g}\left(\mathbb{Z}_{\ell}\right)$. This $\mathrm{Sp}_{2 g}\left(\mathbb{Q}_{\ell}\right)$-action on $\mathcal{A}_{g, \ell}{ }^{\infty}$ induces the $\ell$-adic Hecke correspondences on $\mathcal{A}_{g, N}$.

(2.5) For each $n \in \mathbb{N}$, let $Z(n)=\mathcal{A}_{g, \ell^{n} N} \times_{\mathcal{A}_{g, N}} Z$ and let $Z^{0}(n)$ be the restriction of $Z(n) \rightarrow Z$ to $Z^{0} \subset Z$. The natural map $Z(n) \rightarrow Z$ is a principal étale $\operatorname{Sp}_{2 g}\left(\mathbb{Z} / \ell^{n} \mathbb{Z}\right)$ cover. Moreover the contraction product

$$
Z(n) \times \operatorname{Sp}_{2 g}\left(\mathbb{Z} / \ell^{n} \mathbb{Z}\right), \operatorname{std}\left(\mathbb{Z} / \ell^{n} \mathbb{Z}\right)^{2 g}
$$

of the principal cover $Z(n) \rightarrow Z$ with the standard representation of $\operatorname{Sp}_{2 g}\left(\mathbb{Z} / \ell^{n} \mathbb{Z}\right)$ on $\left(\mathbb{Z} / \ell^{n} \mathbb{Z}\right)^{2 g}$ is isomorphic to the group scheme $A\left[\ell^{n}\right] \rightarrow Z$ of $\ell^{n}$-torsion points of $\underset{\widetilde{Z}}{A} \rightarrow Z$. As $n$ varies the principal covers $Z(n)$ form a projective system; denote by $\widetilde{Z}$ the projective limit of the $Z(n)$ 's; it is a pro-étale principal $\operatorname{Sp}_{2 g}\left(\mathbb{Z}_{\ell}\right)$-cover of $Z$. Similarly denote by $\widetilde{Z^{0}}$ the projective limit of the $Z^{0}(n)$ 's; it is the restriction to $Z^{0}$ of the pro-étale principal $\operatorname{Sp}_{2 g}\left(\mathbb{Z}_{\ell}\right)$-cover $\widetilde{Z} \rightarrow Z$ to $Z^{0}$. Of course $\widetilde{Z}$ is canonically isomorphic to $\mathcal{A}_{g, \ell^{\infty} N} \times \mathcal{A}_{g, N} Z$.

The assumption that $Z$ is stable under the $\ell$-adic Hecke correspondences means that the closed subscheme $\widetilde{Z}$ of $\mathcal{A}_{g, \ell^{\infty} N}$ is stable under the action of $\operatorname{Sp}_{2 g}\left(\mathbb{Q}_{\ell}\right)$ on $\mathcal{A}_{g, \ell^{\infty} N}$. So the action of $\operatorname{Sp}_{2 g}\left(\mathbb{Z}_{\ell}\right)$ on $\widetilde{Z}$ extends to an action of $\operatorname{Sp}_{2 g}\left(\mathbb{Q}_{\ell}\right)$ on $\widetilde{Z}$. This action of $\operatorname{Sp}_{2 g}\left(\mathbb{Q}_{\ell}\right)$ on $\widetilde{Z}$ induces the $\ell$-adic Hecke correspondences on the base scheme $Z$.

(2.6) Choose a point $\tilde{\eta} \in \widetilde{Z}(\bar{\eta})$ above $\eta$; one can write $\tilde{\eta}$ as the limit of a compatible system of points $\tilde{\eta}_{n} \in Z(n)(\bar{\eta})$. Let $Y_{n}$ be the connected component of $Z(n)$ containing $\eta_{n}$. The projective limit $Y$ of the $Y_{n}$ 's is a connected component of $\widetilde{Z}$ and is also a connected component of $\widetilde{Z^{0}}$. According to general Galois theory, the stabilizer in $\mathrm{Sp}_{2 g}\left(\mathbb{Z} / \ell^{n} \mathbb{Z}\right)$ of the connected component $Y_{n}$ is equal to $M_{n}$, and the stabilizer of $Y$ in $\operatorname{Sp}_{2 g}\left(\mathbb{Z}_{\ell}\right)$ is $M$. Also we know from Galois theory that $\operatorname{Aut}\left(Y / Z^{0}\right)=M$ and $Y \times{ }^{M} \mathrm{Sp}_{2 g}\left(\mathbb{Z}_{\ell}\right)=\widetilde{Z^{0}}$. 
The set $\pi_{0}\left(\widetilde{Z^{0}}\right)$ of connected components of $\widetilde{Z^{0}}$ is a pro-finite set, endowed with the pro-finite topology: $\pi_{0}\left(\widetilde{Z^{0}}\right)=\lim \pi_{0}(Z(n))$. The same holds for the set $\pi_{0}(\widetilde{Z})$ of connected components of $\widetilde{Z}$. The assumption that the $\ell$-adic Hecke correspondences operate transitively on $\pi_{0}(Z)$ means that $\operatorname{Sp}_{2 g}\left(\mathbb{Q}_{\ell}\right)$ operates transitively on the set $\pi_{0}(\widetilde{Z})$ of connected components of $\widetilde{Z}$. Clearly $\pi_{0}\left(\widetilde{Z^{0}}\right)$ is a subset of $\pi_{0}(\widetilde{Z})$, and $\pi_{0}(\widetilde{Z})$ is non-canonically homeomorphic to the disjoint union of a finite number of copies of $\pi_{0}\left(\widetilde{Z^{0}}\right)$ because $\operatorname{Sp}_{2 g}\left(\mathbb{Q}_{\ell}\right)$ operates transitively on $\pi_{0}(\widetilde{Z})$. The base point $[Y] \in \pi_{0}\left(\widetilde{Z^{0}}\right)$ gives us a continuous bijection $f_{1}: M \backslash \operatorname{Sp}_{2 g}\left(\mathbb{Z}_{\ell}\right) \stackrel{\sim}{\longrightarrow} \pi_{0}\left(\widetilde{Z^{0}}\right)$ which is necessarily a homeomorphism since the source and the target are both compact.

(2.7) Let $Q$ be the stabilizer subgroup of $Y$ in $\operatorname{Sp}_{2 g}\left(\mathbb{Q}_{\ell}\right)$, consisting of all elements of $\operatorname{Sp}_{2 g}\left(\mathbb{Q}_{\ell}\right)$ which send $Y$ to itself; $Q$ is a closed subgroup of $\operatorname{Sp}_{2 g}\left(\mathbb{Q}_{\ell}\right)$ because the action of $\operatorname{Sp}_{2 g}\left(\mathbb{Q}_{\ell}\right)$ on $\pi_{0}(\widetilde{Z})$ is continuous. By definition, each element of $Q$ operates as an automorphism of $Y ; Q \cap \mathrm{Sp}_{2 g}\left(\mathbb{Z}_{\ell}\right)=M$ because $\operatorname{Aut}\left(Y / Z^{0}\right)=M$. The base point $[Y] \in \pi_{0}(\widetilde{Z})$ gives us a continuous bijection $f_{2}$ from $Q \backslash \operatorname{Sp}_{2 g}\left(\mathbb{Q}_{\ell}\right)$ to $\pi_{0}(\widetilde{Z})$.

(2.8) Lemma The bijection $f_{2}$ above is a homeomorphism.

Proof. The source of $f_{2}$ is locally compact, and is a countable union of compactopen subsets. The target of $f_{2}$ is a profinite topological space, hence is a complete metric space. By Baire's category theorem applied to the complete metric space $\pi_{0}(\widetilde{Z})$, there exists an compact open subset $U$ in $Q \backslash \operatorname{Sp}_{2 g}\left(\mathbb{Q}_{\ell}\right)$ such that $f_{2}(U)$ contains an open subset $W$ of $\pi_{0}(\widetilde{Z})$. Shrinking $W$, we see that there exists a compact open subset $U_{1}$ of $Q \backslash \mathrm{Sp}_{2 g}\left(\mathbb{Q}_{\ell}\right)$ contained in $U$ such that $f_{2}$ induces a homeomorphism from $U_{1}$ to a compact open subset $W_{1}$ of $\pi_{0}(\widetilde{Z})$. Because $f_{2}$ is compatible with the continuous action of $\mathrm{Sp}_{2 g}\left(\mathbb{Q}_{\ell}\right)$ on its source and its target, we conclude that the continuous bijection $f_{2}$ is open, hence it is a homeomorphism.

(2.9) Remark Infinite Galois coverings with action by a non-compact $\ell$-adic Lie group related to Hecke correspondences have been considered in [17], [10], [8] and [7].

\section{$\S 3$. Lemmas}

(3.1) Lemma Let $L$ be a subgroup of finite index of $\operatorname{Sp}_{2 g}\left(\mathbb{Q}_{\ell}\right)$. Then $L=\operatorname{Sp}_{2 g}\left(\mathbb{Q}_{\ell}\right)$.

Proof. This Lemma is surely well-known; here is a proof. Let $T$ be a split maximal torus of $G=\mathrm{Sp}_{2 g}$ over $\mathbb{Q}_{\ell}$. For every root $\alpha$ of $G$, denote by $U_{\alpha}$ the corresponding 
root subgroup. Denote by $\chi_{\alpha}: T \rightarrow \mathbb{G}_{\mathrm{m}}$ the character of $T$ attached to $\alpha$. By assumption $L \cap U_{\alpha}\left(\mathbb{Q}_{\ell}\right)$ is an open subgroup of finite index in $U_{\alpha}\left(\mathbb{Q}_{\ell}\right)$. The $p$-adic group $U_{\alpha}\left(\mathbb{Q}_{\ell}\right)$ is divisible as an abelian group, being the abelian group underlying a vector space over $\mathbb{Q}_{\ell}$. So the subgroup $L \cap U_{\alpha}\left(\mathbb{Q}_{\ell}\right)$ of finite index in $U_{\alpha}\left(\mathbb{Q}_{\ell}\right)$ is equal to $U_{\alpha}\left(\mathbb{Q}_{\ell}\right)$ itself. Since $\operatorname{Sp}_{2 g}\left(\mathbb{Q}_{\ell}\right)$ is generated by the root subgroups $U_{\alpha}\left(\mathbb{Q}_{\ell}\right)$, we have proved that $L=\mathrm{Sp}_{2 g}\left(\mathbb{Q}_{\ell}\right)$.

(3.2) Lemma Let $P$ be a connected closed subgroup of a connected reductive $G$ over $\mathbb{Q}_{\ell}$. Then the coset space $P\left(\mathbb{Q}_{\ell}\right) \backslash G\left(\mathbb{Q}_{\ell}\right)$ is compact if and only if $P$ is a parabolic subgroup of $G$.

Proof. This is a special case of [2, Prop. 9.3].

(3.3) Lemma Let $H$ be a connected semisimple subgroup of $G=\mathrm{Sp}_{2 g}$ over a field $E$ of characteristic 0 . Then the neutral component $\mathrm{N}_{G}(H)^{0}$ of the normalizer of $H$ in $G$ is reductive.

Proof. We offer two proofs. The first one uses compact groups. We may and do assume that $E=\mathbb{C}$. Choose a maximal compact subgroup $K_{H}$ of $H(\mathbb{C})$, and choose a maximal compact subgroup $K_{G}$ of $G(\mathbb{C})$ containing $K_{H}$. From the compact subgroups $K_{H}$ and $K_{G}$ we get compact real forms $H_{\mathbb{R}}$ and $G_{\mathbb{R}}$ of $H$ and $G$ respectively, such that $H_{\mathbb{R}}(\mathbb{R})=K_{H}$ and $G_{\mathbb{R}}(\mathbb{R})=K_{G}$ as subgroups of $G(\mathbb{C})$. Therefore $\mathrm{N}_{G}(H)^{0}$ has a compact real form, namely $\mathrm{N}_{G_{\mathbb{R}}}\left(H_{\mathbb{R}}\right)^{0}$, the neutral component of the normalizer of $H_{\mathbb{R}}$ in $G_{\mathbb{R}}$. Hence $\mathrm{N}_{G}(H)^{0}$ is reductive.

Here is an algebraic proof, due to A. Borel. We may and do assume that $G$ is semisimple. Let $C=\mathrm{Z}_{G}(H)^{0}$ be the neutral component of the centralizer subgroup of $H$, and let $U$ be the unipotent radical of $C$. The group $H$ being reductive, we have natural orthogonal decompositions

$$
\operatorname{Lie}(G)=\operatorname{Lie}(C) \oplus V=\operatorname{Lie}(U) \oplus \operatorname{Lie}(C / U) \oplus V
$$

with respect to the Killing form $B$ on $\operatorname{Lie}(G)$, and each direct summand is stable under the action of $C \cdot H=\mathrm{N}_{G}(H)^{0}$. Since $U$ is unipotent, there is a separated decreasing filtration Fil ${ }^{\bullet}$ of $\operatorname{Lie}(G)$ such that $\operatorname{ad}(u) \cdot \mathrm{Fil}^{i} \subset \mathrm{Fil}^{i+1}$ for all $u \in \operatorname{Lie}(U)$ and all $i$. This implies that the restriction of the Killing form $B$ to $\operatorname{Lie}(U)$ vanishes identically. Therefore $\operatorname{Lie}(U)=(0)$ since $B$ is nondegenerate. Hence $C$ and $\mathrm{N}_{G}(H)^{0}=C \cdot H$ are both reductive.

(3.4) Lemma Assumptions as in $\S 2$. If $A_{\bar{\eta}}$ is not supersingular, then the abelian scheme $A \times{ }_{Z^{0}} Z^{\prime} \rightarrow Z^{\prime}$ is not isogenous to a constant abelian scheme for any finite 
surjective morphism $Z^{\prime} \rightarrow Z$. Conversely if $A_{\bar{\eta}}$ is supersingular, then $A \rightarrow Z^{0}$ is isogenous to a constant abelian scheme.

Proof. Suppose that $A_{\bar{\eta}}$ is not supersingular. Let $\bar{Z}$ be the closure of $Z$ in $\mathcal{A}_{g, N}$. If $A \rightarrow Z^{0}$ is isogenous to an isotrivial abelian scheme after a finite surjective base change $Z^{\prime} \rightarrow Z$, then $\bar{Z}$ is proper. Clearly $\bar{Z}$ is stable under all $\ell$-power Hecke correspondences since $Z$ is. The argument of [3, Prop. 6], which uses [15, Thm. 4.1] and [3, Prop. 1], shows that $\bar{Z}$ contains a supersingular point. This is a contradiction. The converse statement is well-known.

(3.5) Corollary Assumptions as in $\S 2$. Assume moreover that $A_{\bar{\eta}}$ is not supersingular. Then the image $\rho_{\ell}\left(\pi_{1}\left(Z_{x}, \bar{\eta}\right)\right)$ of the $\ell$-adic monodromy representation attached to $A \rightarrow Z^{0}$ is not finite. Conversely, if $A_{\bar{\eta}}$ is supersingular, then $\rho_{\ell}\left(\pi_{1}\left(Z_{x}, \bar{\eta}\right)\right)$ is finite.

Proof. Suppose that $\rho_{\ell}\left(\pi_{1}\left(Z_{x}, \bar{\eta}\right)\right)$ is finite. By [13, Thm. 2.1], there exists a finite surjective base change map $Z^{\prime} \rightarrow Z^{0}$ such that $A \times{ }_{Z^{0}} Z^{\prime} \rightarrow Z$ is isogenous to a constant abelian scheme. This contradicts Lemma 3.4. The converse holds because the geometric $\ell$-adic monodromy group of any abelian scheme isogenous to a constant one is finite.

\section{$\S 4$. Results}

(4.1) Proposition Assumptions as in §2. Assume moreover that the image of the $\ell$-adic monodromy representation $\rho_{A, \ell}$ attached to $A \rightarrow Z^{0}$ is not finite. Then $\rho_{A, \ell}\left(\pi_{1}\left(Z^{0}, \bar{\eta}\right)\right)$ contains an open subgroup of $\operatorname{Sp}\left(\mathrm{T}_{\ell},\langle,\rangle_{\ell}\right)$

Proof. Recall that $G=\operatorname{Sp}\left(V,\langle,\rangle_{\ell}\right)$, identified with $\mathrm{Sp}_{2 g, \mathbb{Q}_{\ell}}$ as an algebraic group over $\mathbb{Q}_{\ell}$. Let $M$ the image of $\pi_{1}\left(Z^{0}, \bar{\eta}\right)$ in $G\left(\mathbb{Q}_{\ell}\right)$.

Let $H$ be the neutral component of the Zariski closure of $M$ in $G ; H$ is not the trivial subgroup by assumption. The closed subgroup $M$ of $\mathrm{Sp}_{2 g}\left(\mathbb{Z}_{\ell}\right)$ is an $\ell$-adic Lie group; denote its Lie algebra by $\mathfrak{m}$. By $[1, \S 7$, Cor. 7.9], the commutator of the Lie algebra of $H$ is contained in the Lie algebra of $M$. Hence it suffices to show that the subgroup $H$ of $G$ is equal to $G$.

Since $Z$ is stable under all $\ell$-power Hecke correspondences, the group $\operatorname{Sp}_{2 g}\left(\mathbb{Q}_{\ell}\right)$ operates naturally on $\widetilde{Z}$. This gives us an action of $\operatorname{Sp}_{2 g}\left(\mathbb{Q}_{\ell}\right)$ on $\widetilde{Z}$, extending the action of $\operatorname{Sp}_{2 g}\left(\mathbb{Z}_{\ell}\right)$ on $\widetilde{Z}$. Notice that this action is not compatible with the projection $\operatorname{map} \widetilde{Z} \rightarrow Z$. 
Recall that $Q$ is the stabilizer subgroup in $\operatorname{Sp}_{2 g}\left(\mathbb{Q}_{\ell}\right)$ of the connected component $Y$ of $\widetilde{Z}$. Although $M$ is not stable under the conjugation action of $Q$, this turns out to hold on the level of Lie algebras. More precisely, for any element $\gamma \in Q$, there exists an open subgroup $U \subset \operatorname{Sp}_{2 g}\left(\mathbb{Z}_{\ell}\right)$ such that $\gamma \cdot U \cdot \gamma^{-1} \subset \operatorname{Sp}_{2 g}\left(\mathbb{Z}_{\ell}\right)$. Therefore $\gamma \cdot(M \cap U) \cdot \gamma^{-1} \subset Q \cap \mathrm{Sp}_{2 g}\left(\mathbb{Z}_{\ell}\right)=M$. Passing to the Lie algebras of the $\ell$-adic Lie groups $M \cap U$ and $M$ yields the desired conclusion that $\operatorname{Ad}(\gamma)(\mathfrak{m}) \subseteq \mathfrak{m}$ for all $\gamma \in Q$.

The fact that the Lie algebra $\mathfrak{m}$ of $M$ is stable under $\operatorname{Ad}(\gamma)$ for all $\gamma \in Q$ implies that the neutral component $H$ of the Zariski closure of $M$ is stable under conjugation by any element $\gamma \in Q$. In other words $Q$ is contained in $\mathrm{N}_{G}(H)\left(\mathbb{Q}_{\ell}\right)$. Since $Q \backslash G\left(\mathbb{Q}_{\ell}\right)$ is compact, we conclude that coset space $\mathrm{N}_{G}(H)^{0}\left(\mathbb{Q}_{\ell}\right) \backslash G\left(\mathbb{Q}_{\ell}\right)$ is compact, where $\mathrm{N}_{G}(H)^{0}$ denotes the neutral component of the algebraic group $\mathrm{N}_{G}(H)$. By Lemma $3.2, \mathrm{~N}_{G}(H)^{0}$ is a parabolic subgroup of $G$.

Now we use a theorem of Grothendieck and a theorem of Deligne in [9, Cor. 1.3.9] and [9, Thm. 3.4.1]. They imply that the neutral component of the Zariski closure of the geometric monodromy group of any pure $\mathbb{Q}_{\ell}$-sheaf over a variety defined over a finite field is semisimple. In our situation Grothendieck's theorem tells us that $H$ is semisimple. Notice that the purity condition comes from the Riemann hypothesis for abelian varieties over finite field, proved by Weil. Since $H$ is semisimple, its normalizer subgroup in $G$ is reductive by Lemma 3.3 .

The subgroup $\mathrm{N}_{G}(H)^{0}$ of $G$ is a parabolic subgroup of $G$ and it is also reductive, hence $\mathrm{N}_{G}(H)^{0}=G$. So $H$ is a non-trivial normal subgroup of $G$. Therefore $H$ is equal to $G$ since $G=\mathrm{Sp}_{2 g}$ is a simple algebraic group over $\mathbb{Q}_{\ell}$.

(4.1.1) Remark According to Cor. 3.5, the assumption that $\rho_{A, \ell}\left(\pi_{1}\left(Z^{0}, \bar{\eta}\right)\right)$ is not finite is equivalent to the condition that $A_{\bar{\eta}}$ is not supersingular.

(4.2) Corollary Assumptions as in $\S 2$. Assume moreover that $\left.A_{\bar{\eta}}\right\}$ is not supersingular. Then $\operatorname{End}\left(A_{\bar{\eta}}\right)=\mathbb{Z}$.

Proof. Obviously the action of $\operatorname{End}\left(A_{\bar{\eta}}\right) \otimes_{\mathbb{Z}} \mathbb{Q}_{\ell}$ on $V=\mathrm{T}_{\ell}\left(A_{\bar{\eta}}\right) \otimes_{\mathbb{Z}_{\ell}} \mathbb{Q}_{\ell}$ commutes with the monodromy representation, hence $\operatorname{End}\left(A_{\bar{\eta}}\right) \otimes_{\mathbb{Z}} \mathbb{Q}_{\ell} \subseteq \mathbb{Q}_{\ell} \cdot \operatorname{Id}_{V}$.

(4.3) Corollary Notation and assumption as in $\S 2$. Suppose that ground field $k$ is $\overline{\mathbb{F}_{p}}$. Then there exists a point $y \in Z\left(\overline{\mathbb{F}_{p}}\right)$ such that $\operatorname{End}_{\overline{\mathbb{F}_{p}}}\left(A_{y}\right) \otimes_{\mathbb{Z}} \mathbb{Q}$ is a number field $E$ with $[E: \mathbb{Q}]=2 g$, and there is exactly one place $v$ in $E$ above $\ell$.

Proof. Use Chebotarev and Prop. 4.1. 
(4.4) Proposition Notation and assumption as in $\S 2$. Assume that $A_{\bar{\eta}}$ is not supersingular. Then the image $\rho_{A, \ell}\left(\pi_{1}\left(Z^{0}, \bar{\eta}\right)\right)$ of the $\ell$-adic monodromy representation of $A \rightarrow Z^{0}$ is equal to $\operatorname{Sp}\left(\mathrm{T}_{\ell},\langle,\rangle_{\ell}\right) \cong \operatorname{Sp}_{2 g}\left(\mathbb{Z}_{\ell}\right)$. Moreover $Z=Z^{0}$, i.e. $Z$ is irreducible.

Proof. We use the notation in the proof of Prop. 4.1. Cor. 3.5 shows that the $\ell$-adic monodromy representation of $A \rightarrow Z^{0}$ is not finite. Prop. 4.1 tells us that the set $\pi_{0}\left(\widetilde{Z}^{0}\right) \cong M \backslash \mathrm{Sp}_{2 g}\left(\mathbb{Z}_{\ell}\right)$ of connected components of $\widetilde{Z^{0}}$ is finite, and so is the set of connected components $\pi_{0}(\widetilde{Z}) \cong Q \backslash \operatorname{Sp}_{2 g}\left(\mathbb{Q}_{\ell}\right)$. Lemma 3.1 implies that $Q=\operatorname{Sp}_{2 g}\left(\mathbb{Q}_{\ell}\right)$. Hence $\widetilde{Z}$ is connected. So the finite set $\pi_{0}\left(M \backslash \operatorname{Sp}_{2 g}\left(\mathbb{Z}_{\ell}\right)\right)=\pi_{0}\left(\widetilde{Z}^{0}\right) \subseteq$ $\pi_{0}(\widetilde{Z})$ has only one element, i.e. $M=\operatorname{Sp}_{2 g}\left(\mathbb{Z}_{\ell}\right)$. Since the map $\widetilde{Z} \rightarrow Z$ is faithfully flat, we deduce that $Z$ is connected.

(4.4.1) Remark We have assumed that the level $N$ is prime to $\ell p$ for simplicity. Our argument still applies if we assume only that the level $N \geq 3$ is prime to $p$. Then Prop. 4.1 remains true, while Prop. 4.4 should be changed to state that the image $\rho_{\ell}\left(\pi_{1}\left(Z^{0}, \bar{\eta}\right)\right)$ of $\rho$ is equal to the principal congruence subgroup of level $N^{\prime}=\left(N, \ell^{\infty}\right)$ in $\mathrm{Sp}_{2 g}\left(\mathbb{Z}_{\ell}\right)$.

(4.5) Let $S$ be a set of prime numbers different from $p$. Then one can consider $S$-adic Hecke correspondences coming from elements of the restricted product $\prod_{\ell \in S}^{\prime} \operatorname{Sp}_{2 g}\left(\mathbb{Q}_{\ell}\right)=: \operatorname{Sp}_{2 g}\left(\mathbb{A}_{\mathbb{Q}, S}\right)$. The method used in the proof of Prop. 4.1, 4.4 applies as well and yield analogous statements. Here we formulate only the case when $S$ is the set of all prime numbers different from $p$. The corresponding Hecke correspondences are usually referred to as the set of all prime-to- $p$ Hecke correspondences.

(4.5.1) Let $W$ be a smooth locally closed subset of $\mathcal{A}_{g, N}$ over $\overline{\mathbb{F}_{p}}$, such that $W$ is stable under all prime-to- $p$ Hecke correspondences and the set of all prime-to- $p$ Hecke correspondences operate transitively on $\pi_{0}(W)$.

(4.5.2) Denote by $\Gamma(N)$ the subgroup of $\operatorname{Sp}_{2 g}\left(\widehat{\mathbb{Z}}^{(p)}\right)=\prod_{\ell \neq p} \operatorname{Sp}_{2 g}\left(\mathbb{Z}_{\ell}\right)$, where $\ell$ runs through all prime numbers different from $p$, consisting of all elements of $\operatorname{Sp}_{2 g}\left(\widehat{\mathbb{Z}}^{(p)}\right)$ which are congruent to Id modulo $N$. 
(4.5.3) Let $W^{0}$ be a connected component of $W$, let $\eta$ be the generic point of $W^{0}$, and let $\bar{\eta}$ be a geometric point above $\eta$. Denote by

$$
\rho_{A, \widehat{\mathbb{Z}}^{(p)}}: \pi_{1}\left(W^{0}, \bar{\eta}\right) \rightarrow \Gamma(N) \subset \operatorname{Sp}_{2 g}\left(\widehat{\mathbb{Z}}^{(p)}\right)
$$

the monodromy representation attached to the prime-to- $p$ torsion points of $A \rightarrow W^{0}$. The following proposition can be proved by the same argument as before; the proof is omitted.

(4.5.4) Proposition Notation as above. Assume that the abelian variety $A_{\bar{\eta}}$ is not supersingular. Then the image of the monodromy representation $\rho_{A, \widehat{\mathbb{Z}}^{(p)}}$ is equal to $\Gamma(N)$. Moreover $W=W^{0}$, i.e. $W$ is irreducible.

Remark We shall prove in Prop. 4.6 a sort of "independence of $\ell$ " result, to the effect that every subvariety $Z$ in $\mathcal{A}_{g, N}$ which is stable under all $\ell$-adic Hecke correspondences is actually stable under the action of all prime-to- $p$ Hecke correspondences. Therefore Prop. 4.5.4 applies, giving the maximality of the Galois representation attached to the prime-to- $p$ torsion points of $A \rightarrow Z$.

(4.6) Proposition Notation and assumptions as above. In particular, $Z$ is a smooth irreducible subvariety of $\mathcal{A}_{g, N}$ stable under all $\ell$-adic Hecke correspondences, and $A_{\bar{\eta}}$ is not supersingular. Then $Z$ is stable under the action of all $\lambda$-adic Hecke correspondences for any prime number $\lambda \neq p$.

Proof. The idea of the proof already appeared on page 477 of [3]; here we explain the use of the strong approximation theorem in more detail, correcting some inaccuracies in loc. cit. We may and do assume that $g \geq 2$. As in the proof of Lemma 3.4, the Zariski closure $\bar{Z}$ of $Z$ contains a supersingular point $s$. Denote by $U$ (resp. $G$ ) the unitary group (resp. the special unitary group) over $\mathbb{Q}$ attached to the semisimple algebra $\operatorname{End}\left(A_{s}\right) \otimes_{\mathbb{Z}} \mathbb{Q}$ with the Rosati involution. The group $G$ is an inner form of $\mathrm{Sp}_{2 g}$ such that $G(\mathbb{R})$ is compact, while $G\left(\mathbb{Q}_{r}\right)$ is non-compact for every prime number $r$, including $r=p$. For every prime number $r \neq p$, the groups $G$ is split over $\mathbb{Q}_{r}$. Moreover, if $r \neq p$, then the $\mathbb{Z}_{r}$-lattice $\operatorname{End}\left(A_{s}\right) \otimes_{\mathbb{Z}} \mathbb{Z}_{r}$ in $\operatorname{End}\left(A_{s}\right) \otimes_{\mathbb{Z}} \mathbb{Q}_{r}$ defines a hyperspecial maximal compact subgroups $G\left(\mathbb{Z}_{r}\right)$ of $G\left(\mathbb{Q}_{r}\right)$, isomorphic to $\mathrm{Sp}_{2 g}\left(\mathbb{Z}_{r}\right)$. The isomorphism is unique up to conjugation by elements of $\mathrm{Sp}_{2 g}\left(\mathbb{Z}_{r}\right)$.

Let $U\left(\mathbb{Z}_{p}\right):=U\left(\mathbb{Q}_{p}\right) \cap\left(\operatorname{End}\left(A_{s}\right) \otimes_{\mathbb{Z}} \mathbb{Z}_{p}\right)^{\times}, G\left(\mathbb{Z}_{p}\right):=G\left(\mathbb{Q}_{p}\right) \cap\left(\operatorname{End}\left(A_{s}\right) \otimes_{\mathbb{Z}} \mathbb{Z}_{p}\right)^{\times}$. We note that $\operatorname{End}\left(A_{s}\right) \otimes_{\mathbb{Z}} \mathbb{Z}_{p} \stackrel{\sim}{\rightarrow} \operatorname{End}\left(A_{s}\left[p^{\infty}\right]\right)$ by Tate's theorem in [18], because there exists a finite extension $\mathbb{F}_{q}$ of $\mathbb{F}_{p}$ with $q=p^{2 a}, a \in \mathbb{N}$, such that the principally polarized abelian variety $A_{s}$ is defined over $\mathbb{F}_{q}$, and the Frobenius morphism $\mathrm{Fr}_{q}$ is equal to $p^{a} \cdot$ Id. So we can identify $U\left(\mathbb{Z}_{p}\right)$ with $\operatorname{Aut}\left(\operatorname{End}\left(A_{s}\left[p^{\infty}\right], *\right)\right)$. The latter 
group operates naturally on the local deformation space $\mathcal{A}_{g, N}^{/ s}$, and is sometimes referred to as the local automorphism group of the deformation space.

For each prime number $r \neq p$, let $\Gamma_{r}=G\left(\mathbb{Z}_{r}\right)$ if $(r, N)=1$, and let $\Gamma_{r}$ be the principal congruence subgroup of level $r^{e}$ if $r^{e} \| N, e \geq 1$. According to the strong approximation theorem, the image of

$$
G(\mathbb{Q}) \cap\left(\Gamma_{p} \times \prod_{r \neq p, \ell} \Gamma_{r}\right)
$$

in $G\left(\mathbb{Z}_{p}\right)$ is dense in $G\left(\mathbb{Z}_{p}\right)$. This implies that the formal completion $\bar{Z}^{/ s}$ of $\bar{Z}$ at $s$, as a closed formal subscheme of $\mathcal{A}_{g, N}^{/ s}$, is stable under the action of an $G\left(\mathbb{Z}_{p}\right)$. This is an example of a general principle underlying the methods used in [3]; it can be seen as follows. For any element $\gamma_{p}$ in $G\left(\mathbb{Z}_{p}\right)$ and any compact open subgroup $K_{m}$ of $G\left(\mathbb{Z}_{p}\right)$, there exists an element $u \in G(\mathbb{Q})$ such that $u_{r} \in \Gamma_{r}$ for every prime number $r \neq p, \ell$, and $u_{p} \cdot \gamma_{p} \in K_{m}$ in $G\left(\mathbb{Z}_{p}\right)$. Here $u_{r}$ and $u_{p}$ denote the image of $u$ in $G\left(\mathbb{Q}_{r}\right)$ and $G\left(\mathbb{Q}_{p}\right)$ respectively. Let $u_{\ell}$ be the image of $u$ in $G\left(\mathbb{Q}_{\ell}\right)$. Then the action on $\mathcal{A}_{g, N}^{/ s}$ of the Hecke correspondence $\mathcal{H}\left(u_{\ell}\right)$ attached to $u_{\ell}$ is equal to the natural action of $u_{p}^{-1}$ on the local deformation space $\mathcal{A}_{g, N}^{/ s}$. For every natural number $m \geq 1$, one can choose $K_{m}$ sufficiently small so that the actions of $\gamma_{p}$ and $u_{p}^{-1}$ on $\mathcal{A}_{g, N}^{/ s}$ coincide on the $m$-th infinitesimal neighborhood $\operatorname{Spec}\left(\mathcal{O}_{s, \mathcal{A}_{g, N}} / \mathfrak{m}_{s, \mathcal{A}_{g, N}}^{m}\right)$ of $s$ in $\mathcal{A}_{g, N}$. Since $\bar{Z}$ is stable under the $\ell$-adic Hecke correspondence $\mathcal{H}\left(u_{\ell}\right)$, the $m$-th infinitesimal neighborhood $\operatorname{Spec}\left(\mathcal{O}_{\bar{Z}_{s}} / \mathfrak{m}_{s, \bar{Z}}^{m}\right)$ of $s$ in $\bar{Z}$, as a closed subscheme $\operatorname{Spec}\left(\mathcal{O}_{s, \mathcal{A}_{g, N}} / \mathfrak{m}_{s, \mathcal{A}_{g, N}}^{m}\right)$, is stable under the action of $\gamma_{p}$, for every natural number $m \geq 1$. Therefore the closed formal subscheme $\bar{Z}^{/ s}$ of $\mathcal{A}_{g, N}^{/ s}$ is stable under the action of $\gamma_{p}$.

We may and do assume that $\lambda \neq \ell$. Let $\gamma_{\lambda}$ be an element of $G\left(\mathbb{Q}_{\lambda}\right)$. Let $W$ be the image of $\bar{Z}$ under the Hecke correspondence $\mathcal{H}\left(\gamma_{\lambda}\right)$ attached to $\gamma_{\lambda}$. According to the strong approximation theorem, the image of $G(\mathbb{Q})$ in the restricted product $\prod_{r \neq \lambda}^{\prime} G\left(\mathbb{Z}_{r}\right)$ is dense. So there exists an element $\delta$ in $G(\mathbb{Q})$, such that

- $\delta_{r} \in \Gamma_{r}$ for all prime number $r \neq \ell, \lambda$,

- $\delta_{\lambda} \in\left(\gamma_{\lambda} \cdot \Gamma_{\lambda}\right) \cap\left(\Gamma_{\lambda} \cdot \gamma_{\lambda}\right)$, where $\delta_{r}$ and $\delta_{\lambda}$ denote the image of $\delta$ in $G\left(\mathbb{Q}_{r}\right)$ and $G\left(\mathbb{Q}_{\lambda}\right)$ respectively.

Then the effect on $\mathcal{A}_{g, N}^{/ s}$ of the Hecke correspondence $\mathcal{H}\left(\gamma_{\lambda}\right)$ attached to $\gamma_{\lambda}$ is equal to the composition of the action $\delta_{p}^{-1}$ and the Hecke correspondence $\mathcal{H}\left(\delta_{\ell}^{-1}\right)$ attached 
to $\delta_{\ell}^{-1}$. Since $\bar{Z}^{/ s}$ is invariant under the action of $G\left(\mathbb{Z}_{p}\right)$ and $Z$ is stable under all $\ell$ adic Hecke correspondences, we deduce that $W$ contains $s$ and the formal completion $W^{/ s}$ coincide with $Z^{/ s}$. Notice that the same statement holds if $s$ is replaced by any supersingular point $s_{1}$ in $\bar{Z}$.

The prime-to- $p$ Hecke orbit $\mathcal{H}_{\text {non-p }} \cdot s$ of $s$ in $\mathcal{A}_{g, N}$, induced by elements of the symplectic group, is in natural bijection with the finite set

$$
\left(G(\mathbb{Q}) \cap G\left(\mathbb{Z}_{p}\right)\right) \backslash \prod_{r \neq p}^{\prime} G\left(\mathbb{Q}_{r}\right) / \Gamma(N)
$$

where $\Gamma(N)$ is the principle congruence subgroup of $\prod_{r \neq p}^{\prime} G\left(\mathbb{Q}_{r}\right)$ of level $N$. By the strong approximation theorem, the $\ell$-adic Hecke correspondences induced by elements of $\operatorname{Sp}_{2 g}\left(\mathbb{Q}_{\ell}\right) \cong G\left(\mathbb{Q}_{\ell}\right)$ operates transitively on $\mathcal{H}_{\text {non-p }} \cdot s$. It is clear that the subvariety $W$ of $\mathcal{A}_{g, N}$, being the image of the irreducible subvariety $\bar{Z}$ under the Hecke correspondence $\mathcal{H}\left(\gamma_{\lambda}\right)$, has the property that each of its irreducible component contains a point of $\mathcal{H}_{\text {non-p }} \cdot s$. The conclusion of the previous paragraph, applied to points of the Hecke orbit $\mathcal{H}_{\text {non-p }} \cdot s$ of $s$, implies that $W$ is contained in and hence equal to $\bar{Z}$. We have shown that $\bar{Z}$ is stable under all Hecke correspondences attached to elements of $\operatorname{Sp}_{2 g}\left(\mathbb{Q}_{\lambda}\right)$.

(4.7) Remark The irreducibility results in 4.4 and 4.5.4 is handy for proving the irreducibility of certain subsets of $\mathcal{A}_{g, N}$ defined by geometric invariants which are invariant under prime-to- $p$ isogenies. An example is a result of Oort on the set of irreducibility of any non-supersingular Newton polygon stratum in $\mathcal{A}_{g, N}$. We sketch the argument here; see [16] for more details. Let $W_{\xi}$ be a Newton polygon stratum in $\mathcal{A}_{g, N}$ which is not supersingular. It is known that $W_{\xi}$ is equi-dimensional, and the closure of every irreducible component of $W_{\xi}$ contains an irreducible component of the supersingular locus of $\mathcal{A}_{g, N}$; see [12], [11]. One can verify that the primeto- $p$ Hecke correspondences operate transitively on the irreducible components of the supersingular locus, using the description of the supersingular locus in [12]. Furthermore local information is available from deformation theory at points of a dense open subset of the supersingular locus, see [14]. A standard degeneration method in algebraic geometry enables one to deduce from adequate local information that the set of all prime-to- $p$ Hecke correspondences operate transitively on the irreducible components of $W_{\xi}$. Then one can apply Prop. 4.5.4 to conclude that $W_{\xi}$ is irreducible.

(4.8) Remark One can deduce the irreducibility of non-supersingular central leaves from the irreducibility of non-supersingular Newton polygon strata, using Prop. 4.4 and the notation of hypersymmetric points in [5], [6]. As the last example, C.-F. Yu 
has proved the irreducibility of non-supersingular central leaves in a Hilbert modular variety using Prop. 4.4.

\section{References}

[1] A. Borel. Linear Algebraic Groups. (Notes taken by Hyman Bass.) Benjamin, 1969.

[2] A. Borel and J. Tits. Groupes Réductifs. Publ. Math. IHES., 27:55-150, 1965.

[3] C.-L. Chai. Every ordinary symplectic isogeny class in positive characteristic is dense in the moduli. Invent. Math., 121:439-479, 1995.

[4] C.-L. Chai. Hecke orbits on Siegel modular varieties. In Geometric Methods in Algebra and Number Theory, Progress in Mathematics 235, Birkhäuser, pp. 71-107, 2004

[5] C.-L. Chai. Hypersymmetric abelian varieties. Preprint, 6 pages. Available from http://www. math. upenn. edu/ ${ }^{\sim}$ chai

[6] C.-L. Chai and F. Oort. Hypersymmetric abelian varieties. To appear in the Proceedings of a conference on the occasion of the 60th birthday of John Coates, a special issue of Quarterly J. Pure. Applied Math. Available from http://www. math. upenn. edu/ ${ }^{\text {chai }}$

[7] I. V. Cerednik. Uniformization of algebraic curves by discrete arithmetic subgroups of PGL $q_{2}\left(k_{w}\right)$ with compact quotient spaces. Math. USSR-Sb., 29:55-76, 1976.

[8] P. Deligne. Traveaux de Shimura. Sém. Bourbaki, 1970/71, no. 389. In LNM 244, Springer-Verlag, pp. 123-165, 1971.

[9] P. Deligne. La conjecture de Weil. II. Publ. Math. IHES., 58:138-252, 1980.

[10] Y. Ihara. On Congruence Monodromy Problems, vol. I, II. Lecture Notes no. 1, 2, Department of Mathematics, University of Tokyo, 1968, 1969.

[11] A. J. de Jong and F. Oort. Purity and the stratification by Newton polygons. J. Amer. Math. Soc., 13:209-241, 2000.

[12] K. Z. Li and F. Oort. Moduli of Supersingular Abelian Varieties. LNM 1680, Springer-Verlag, 1998.

[13] F. Oort. Subvarieties of moduli spaces. Invent. Math., 24:95-119, 1974. 
[14] F. Oort. Newton polygon and formal groups: conjectures by Manin and Grothendieck. Ann. Math., 152:183-206, 2000.

[15] F. Oort. A stratification of a moduli space of polarized abelian varieties. In Moduli of Abelian Varieties, Progress in Math. 195, Birkhäuser, pp. 345-416, 2001.

[16] F. Oort. Monodromy, Hecke orbits and Newton polygon strata. Available from http://www.math.uu.nl/people/oort. Informal notes of a lecture given in Bonn on Feb. 24, 2003.

[17] I. I. Pjateckii-Shapiro and I. R. Shafarevich. Galois theory of transcendental extensions and uniformization. Izv. Akad. Nauk SSSR Ser. Math., 30:671-704, 1966.

[18] J. Tate. Endomorphisms of abelian varieties over finite fields. Invent. Math., 2:134-144,1966.

Ching-Li Chai

Department of Mathematics

University of Pennsylvania

Philadelphia, PA 19003

U. S. A.

E-mail:chai@math.upenn.edu

Home page: http://www.math.upenn.edu/ ${ }^{\sim}$ chai 